Japan. J. Med. Sci. Biol., 20, 243-249, 1967

\title{
ON A NEW LUNG FLUKE, PARAGONIMUS BANGKOKENSIS sp. nOv. IN THAILAND (TRAMATODA : TROGLOTREMATIDAE)
}

\author{
ICHIRO MIYAZAKI* AND SUVAJRA VAJRASTHIRA** \\ * Department of Parasitology, Faculty of Medicine, \\ Kyushu University, Fukuoka, Japan \\ ** Department of Helminthology, Faculty of Tropical Medicine, \\ University of Medical Sciences, Bangkok, Thailand \\ (Received: February 1st, 1967)
}

\begin{abstract}
SUMMARY : Paragonimus bangkokensis sp. nov. was found in Sarika village, Nakorn-nayok province, Central Thailand, known as an endemic area of human paragonimiasis and situated about $90 \mathrm{~km}$ from Bangkok. The metacercariae were abundant in the liver and muscle of a crab, Potamon smithianus Rathbun, and matured experimentally in a cat and a bandicoot, Bandicota indica (Bechstein). In addition, adults of the new species were found in the lungs of two out of five small Indian mongooses, Herpestes javanicus Geoffroy captured in the same locality. As the crab host is frequently eaten uncooked by inhabitants of the area, there is a possibili $y$ of human infection by the new fluke.
\end{abstract}

\section{INTRODUCTION}

In Thailand three cases of human paragonimiasis and one endemic area of the disease in Saraburi province were recorded by Prommas (1928), Harinasuta, Kruatrachue and Tandhanand (1957), Sirisumpan (1963) and Vajrasthira, Harinasuta and Maiphoom (1959) respectively, but the lung fluke itself was not found in either adult or larval stage. In 1964 Daengsvang, Papasarathorn and Tongkoom first recognized adults of Paragonimus westermani (Kerbert, 1878) from two leopards, which were captured in Chumporn province, South Thailand. Then, Miyazaki and Wykoff (1965) discovered another adult lung fluke from cats and its metacercaria from a crab, Parathelphusa germaini (Rathbun) in Udornthani province, Northeast Thailand, and they proposed a new specific name, Paragonimus siamensis, for this worm. In 1966 Vajrasthira et al. reported the second endemic area of paragonimiasis in Nakorn-nayok province adjacent to the above-mentioned Saraburi, Central Thailand. They found eggs of Paragonimus in sputum of 33 patients out of 503 inhabitants with pulmonary symptoms.

Since 1965 the present authors have been continuing co-operative researches on the taxonomy of Paragonimus in the new endemic area in Nakorn-nayok situated about 90 $\mathrm{km}$ from Bangkok. Miyazaki and Vajrasthira (1967) not only confirmed the existence of $P$. westermani and $P$. siamensis, but also first recognized $P$. heterotremus Chen et Hsia, 1964 in that area, which had been found originally in the mainland of China. Furthermore, Miyazaki and Harinasuta (1966) first reported two immature worms of this

* 宮崎一郎 (九州大学医学部寄生虫学教室) 
lung fluke from a 13-year-old boy living in the same area. The authors revealed that $P$. heterotremus was one of the causative agents of human paragonimiasis, and supposed that this fluke presumably played an important role in the endemic area of Nakorn-nayok.

In this paper the authors wish to describe another lung fluke, as the fourth species of the genus Paragonimus in Thailand, and to propose the new specific name, Paragonimus bangkokensis sp. nov. for the Capital of Thailand located not far from the type locality of this lung fluke.

\section{MATERIALS AND MethodS}

Metacercariae belonging to the genus Paragonimus were abundant in the liver and the muscle of a crab, Potamon (Potamon) smithianus Rathbun, 1923 captured in a stream running through Sarika village, Nakorn-nayok province. Some of the metacercariae obtained were investigated morphologically and others were experimentally fed to a cat and a bandicoot, Bandicota indica (Bechstein). Mature worms obtained from the mammalian hosts were flattened in alcohol, stained with carmine and fast green, and mounted in balsam. On the other hand, five small Indian mongooses, Herpestes javanicus Geoffroy captured in the above-mentioned village were killed, and two of them were found to harbor one and three mature lung flukes, respectively. These worms, when flattened, stained and mounted in balsam, completely agreed morphologically with those which were obtained experimentally.

\section{DESCRIPTION}

\section{Paragonimus bangkokensis sp. nov. *}

\section{Adult}

Four specimens used in the present description were obtained from the experimentally infected cat. The worms were fully matured, containing many eggs in the uterus. The following measurements are in millimeters.

Holotype (Fig. 1) 13.9 long and 5.0 wide. Whole body covered with cuticular spines arranged in groups (Fig. 3). Oral sucker 0.81 by 0.25 ; ventral sucker 0.81 by 0.77 . Ovary 1.56 by 1.02 in outline, situated on the left side and somewhat posterior to the ventral sucker, showing delicate branching. Left and right testis 1.75 by 0.91 and 1.25 by 0.82 in outline, respectively. Branching of testes much more simple than that of ovary (Fig. 8). Uterus situated on opposite side to ovary contains numerous eggs, yellowish in color and provided with uniform shell, having no thickening on the Enon-operculated pole. Vitelline gland widely distributed on both sides of body. Excretory bladder extends to bifurcation of intestine ; ceca extend to posterior end of body.

Three paratypes show similar features to the holotype in every respect, except that the ovary is situated on the right side in two of them (Figs. 2 and 9). Measurements of the paratypes are summarized in Table 1.

\section{Metacercaria}

The following measurements are in micra. The metacercaria spherical in shape and provided with the outer cyst and the inner cyst, space between them being very variable (Fig. 4). Wall of the outer cyst thin and fragile, and that of the inner cyst also thin

* 和名：バンュックハイキュウチュウ 

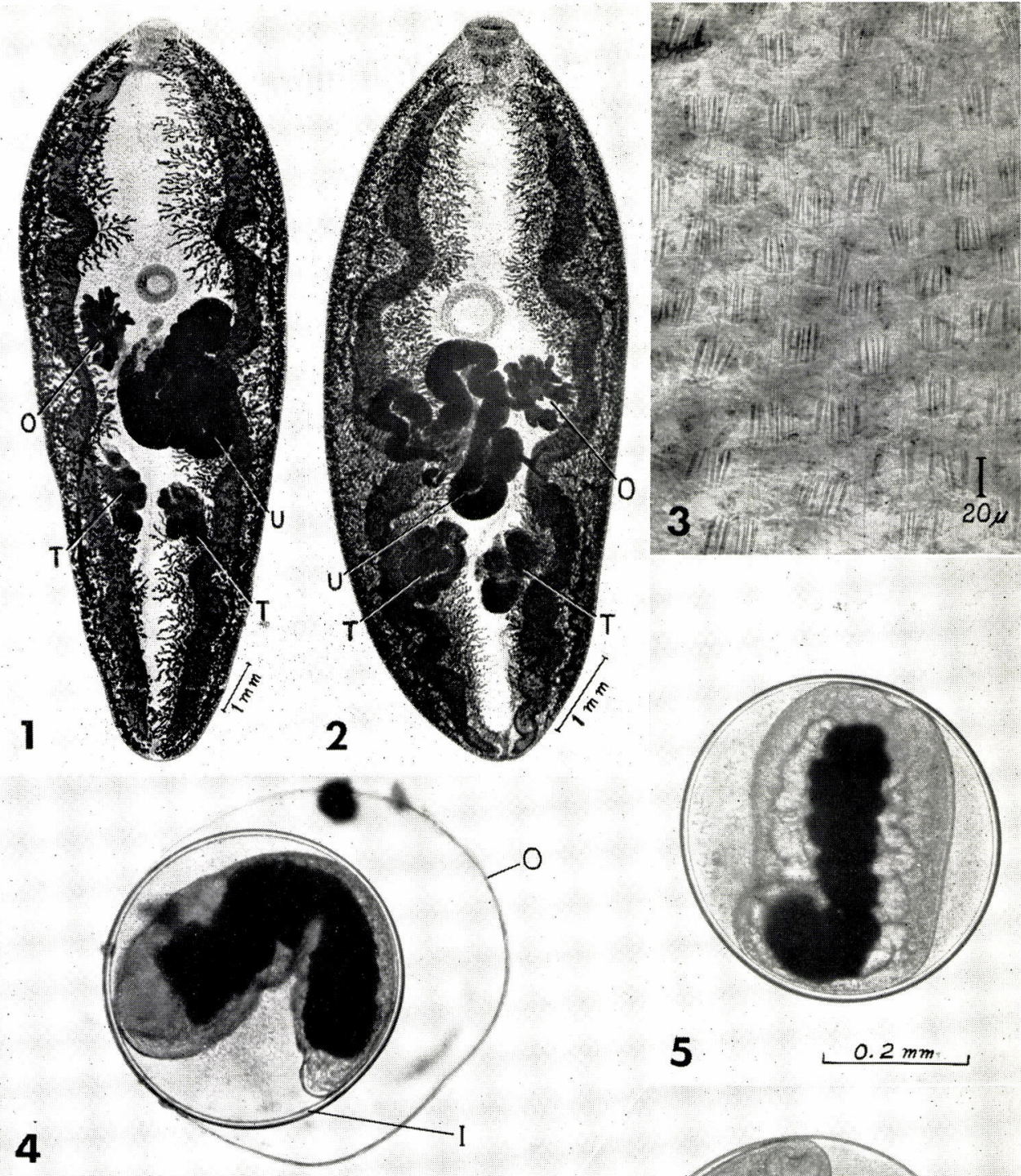

6
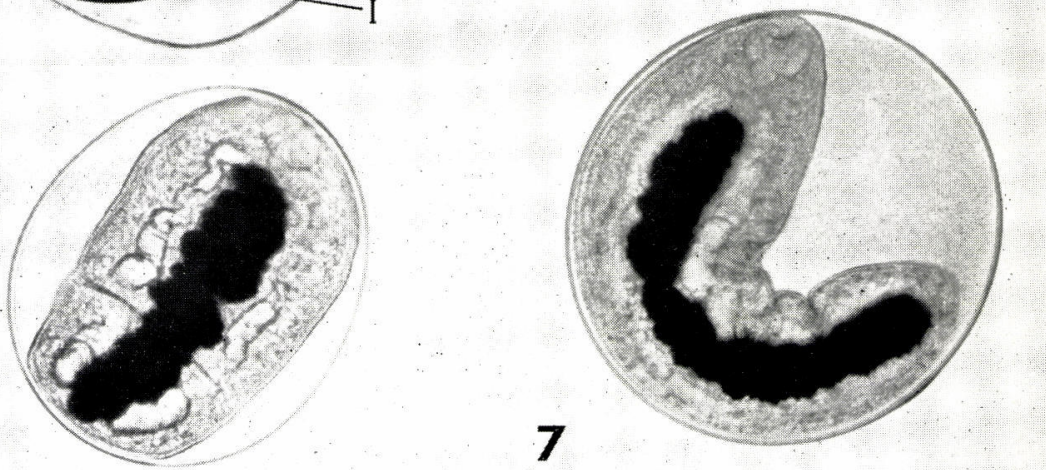

Figs. 1-3. Stained specimens of two adult $P$. bangkokensis. (O) ovary, $\mathrm{T}$ : testis, $\mathrm{U}$ : uterus.) 1. Holotype, dorsal view. 2. Paratype No. 2, ventral view. 3. Cuticular spines on the dorso-anterior part of the holotype.

Figs. 4-7. Four living metacercariae of $P$. bangkokensis under the same magnification, free of pressure except Fig. 7. (I : inner cyst, O : outer cyst.) Figs. 5-7 lack the fragile outer cyst. Note fine granules in the inner cyst and winding of intestines. 
Table 1. Measurements of three paratypes. (in $\mathrm{mm}$ )

\begin{tabular}{ccccccc}
\hline No. & Body & Oral sucker & Ventral sucker & Ovary & Left testis & Right testis \\
\hline 1 & $9.1 \times 3.2$ & $0.59 \times 0.46$ & $0.74 \times 0.78$ & $1.09 \times 0.62$ & $0.91 \times 0.73$ & $1.00 \times 0.64$ \\
2 & $8.5 \times 3.5$ & $0.67 \times 0.43$ & $0.78 \times 0.77$ & $0.91 \times 0.73$ & $1.00 \times 0.65$ & $1.00 \times 0.73$ \\
3 & $8.6 \times 3.1$ & $0.63 \times 0.36$ & $0.75 \times 0.73$ & $0.82 \times 0.76$ & $0.80 \times 0.69$ & $1.00 \times 0.65$ \\
\hline
\end{tabular}

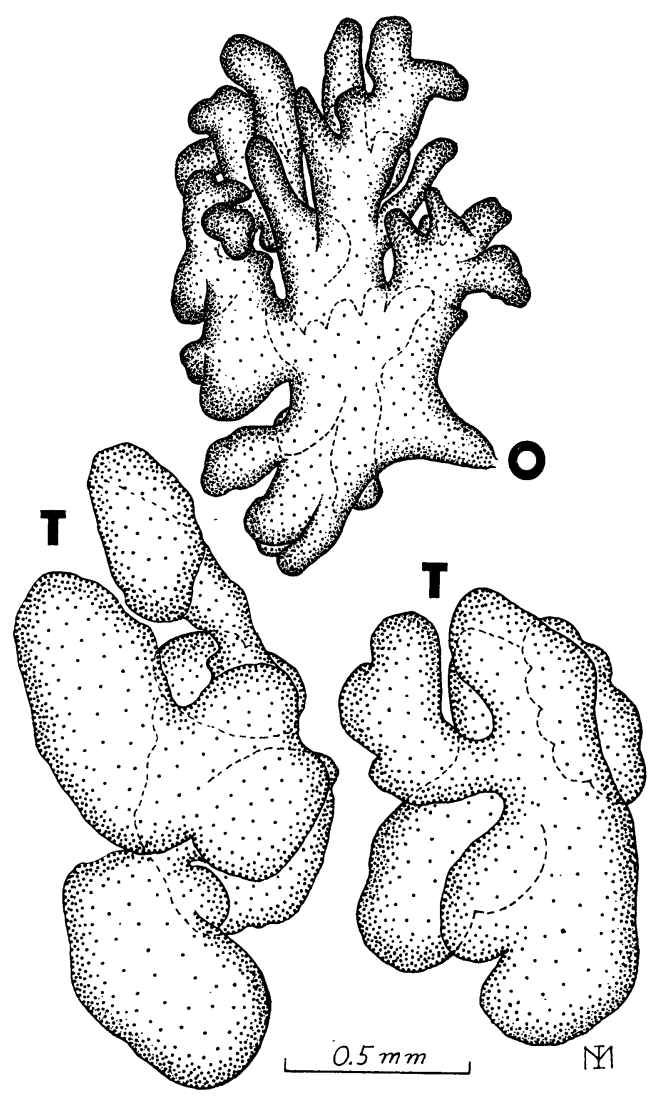

Fig. 8. Detail of ovary $(\mathrm{O})$ and testes $(\mathrm{T})$ of the holotype under the same magnification, dorsal view.

but elastic and stronger, measuring 12-8 (aver. 10) in thickness. Diameter of the inner cyst $476-405$ by $428-357$ (aver. 435 by 398 ), when free of pressure. Because there is large space between the larva and the inner cyst, the former can move freely and the latter is sometimes slightly changed in form. Most of the larvae contain numerous pinkish globules within the body, showing very beautiful appearance, and pale yellowish granules are usually recognized within the inner cyst (Figs. 4-7). Larval body densely armed with single spines all over, and provided with two suckers of almost the same size, a long excretory bladder and two intestines. A small stylet sometimes seen in the oral sucker. Excretory bladder extends to bifurcation of intestine, which runs along the bladder to posterior end of body, showing character- istically fine winding (Fig. 10). 


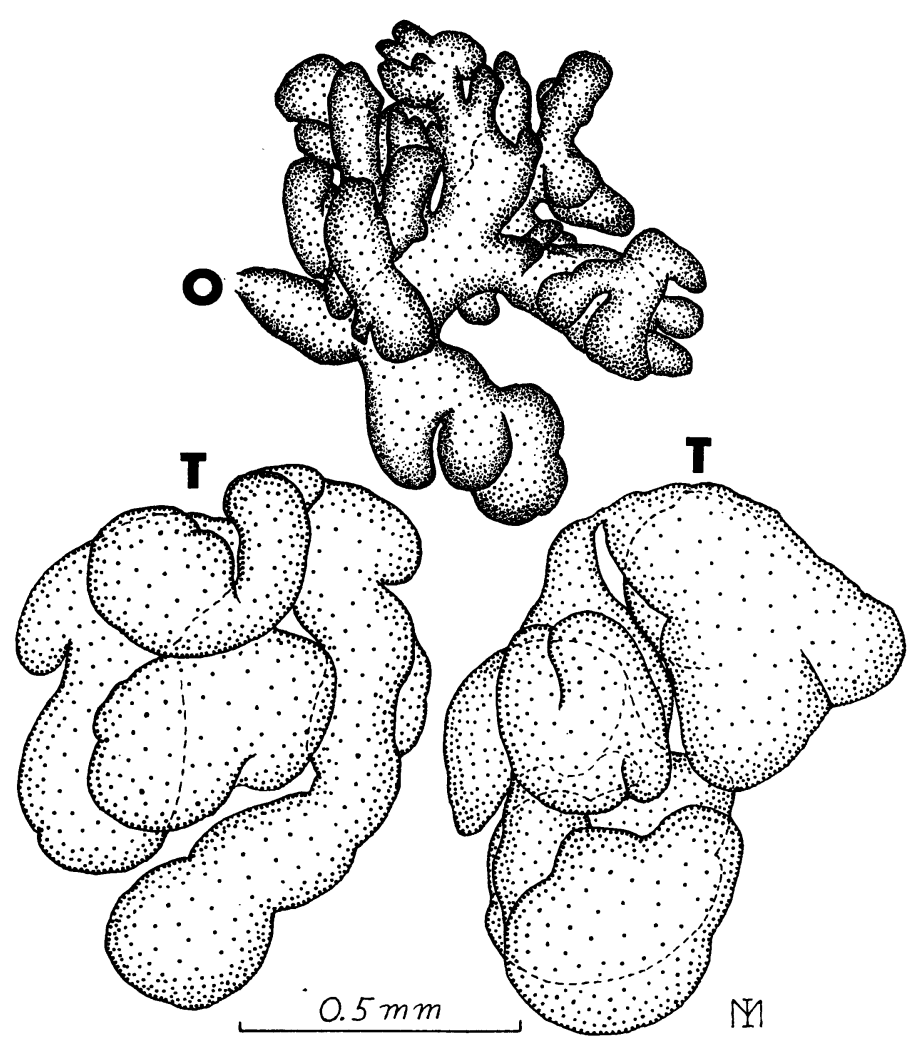

Fig. 9. Detail of ovary $(\mathrm{O})$ and testes $(\mathrm{T})$ of the paratype No. 2 under the same magnification, ventral view.

Two excysted larvae were fixed in formalin under slight cover-glass pressure. One larva measures 881 by 359 in body size; oral sucker 97 by 75 and ventral sucker 94 by 92 . The other one 992 by 370 in size; oral and ventral sucker 103 by 67 and 101 by 99 , respectively.

Host: Felis catus Linne (experimental), Herpestes javanicus Geoffroy (natural).

Location: Lungs.

Locality: Sarika village, Nakorn-nayok province, Thailand.

Second intermediate host: Potamon smithianus Rathbun.

Type specimens: Department of Parasitology, Faculty of Medicine, Kyushu University, Fukuoka, Japan.

\section{DisCUSSION}

Twenty-six species of Paragonimus were hitherto reported in literature. But there are many confusions in taxonomy, and 17 species are regarded as valid ones at present, of which adults of $P$. bangkokensis are most similar to those of $P$. skrjabini Chen, 1959 for the following reasons: 1) the body is slender, 2) the oral sucker is somewhat smaller than the ventral sucker, 3) the ovary is delicately branched and nearly the same in size with the testes, and 4) the testes are more simply branched than the ovary. 


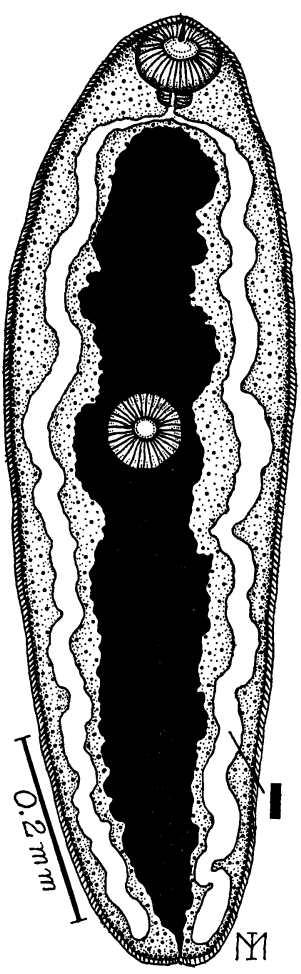

Fig. 10. Excysted living larva of $P$. bangkokensis under slight cover-glass pressure. ( $\mathrm{I}$ : intestine) Note fine winding of intestines. Rim of the ventral sucker is partly covered by granules in the excretory bladder.

But, the two species are easily differentiated by the arrangement of cuticular spines: those of $P$. skrjabini are singly spaced instead of being grouped like those of $P$. bangkokensis. Moreover, the metacercariae of the two species are quite different: those of $P$. skrjabini are a little smaller and the inner cyst wall is thicker than those of $P$. bangkokensis. In addition, the larvae of the former completely fill the space of the inner cyst strongly contracting the body. This appearance resembles the metacercariae of $P$. westermani or $P$. miyazakii Kamo et al., 1961, and is quite different from those of the new species. On the other hand, almost the same size of the oral and ventral suckers and fine winding of the intestines are characteristic of the larvae of $P$. langkokensis, which cannot be seen in other species except one, Euparagonimus cenocopiosus Chen, 1962. But in the latter species the excretory bladder does not extend to the bifurcation of the intestine, ending at the level of the ventral sucker. This is a clearcut difference from the new species.

Incidentally, the new lung fluke has never been found in humans, but medical attention must be paid to the fluke, because its crab host, Potamon smithianus is frequently eaten uncooked by inhabitants. It seems, however, very difficult to identify the new species by the shape of eggs found in sputum of patients, as these eggs show no characteristic features distinguishable from other species of the genus. 


\section{ACKNOWLEDGEMENTS}

The authors wish to express their sincere thanks to Prof. C. Harinasuta, Dean of the Faculty of Tropical Medicine, University of Medical Sciences, for his encouragement and support of this study, and to Prof. S. Miyake of the Faculty of Agriculture, Kyushu University for his identification of the crab host. This work was supported in part by a Scientific Research Grant from the Ministry of Education, Japan.

\section{REFERENCES}

CHEN, H. T. (1960): Taxonomic consideration of Paragonimus, including morphological notes on P. skrjabini Chen. Acta Zool. Sinica, 12, 27-36. (Text in Chinese with English summary)

CHEN, H. T. (1964): The Chinese species of paragonimid trematodes, their phylogenetic relationship and faunal distribution. Acta Parasit. Sinica, 1, 53-66. (Text in Chinese with English summary)

Daengsvang, S., Papasarathorn, T. AND Tongkoom, B. (1964): Paragonimus westermani (Kerbert, 1878) in Thai leopards. Ann. Trop. Med. Parasit., 58, 304-306.

Harinasuta, T., KRUATRACHUE, M. AND TANDhanand, S. (1957) : A case of paragonimiasis in Thailand. J. Med. Ass. Thai., 40, 233-234.

MrYAZAKI, I. AND HARINASUTA, T. (1966): The first case of human paragonimiasis caused by Paragonimus heterotremus Chen et Hsia, 1964. Ann. Trop. Med. Parasit., 60, 509-514.

MIYAZAKI, I. AND VAJRASTHIRA, S. (1967): Occurrence of the lung fluke Paragonimus heterotremus Chen et Hsia, 1964 in Thailand. (Research note.) J. Parasit., 53, 207.

MrYaZAKI, I. AND WyKoff, D. E. (1965): On a new lung fluke Paragonimus siamensis n. sp. found in Thailand (Trematoda: Troglotrematidae). Japan. J. Parasit., 14, 251-257.

Prommas, C. (1928): Paragonimiasis, opisthorchiasis and Madura foot. J. Med. Ass. Thai., $11,67$.

SIRISUMPAN, P. (1963): A case of pulmonary paragonimiasis occurring in a new area in Thailand. J. Med. Ass. Thai., 46, 212.

VAJRASTHIRA, S., HARINASUTA, C. AND MAIPHOOM, C. (1959): Study on helminthic infections in Thailand. 2. The incidence of paragonimiasis in the first recognized endemic area. Japan. J. Exp. Med., 29, 159-166.

Vajrasthira, S., Setasubun, P., Radomyos, P. ANd Harinasuta, C. (1966): Paragonimiasis investigations in Thailand with special reference to epidemiology. Abstr. 2nd Med. Conf. Parasit. Dis., Bangkok, 57-58. 